\title{
Elementary Polynomial Identities Involving $q$-Trinomial Coefficients
}

To George E. Andrews, on the occasion of his 80th birthday

\author{
Alexander Berkovich and Ali Kemal Uncu
}

\begin{abstract}
We use the $q$-binomial theorem to prove three new polynomial identities involving $q$-trinomial coefficients. We then use summation formulas for the $q$-trinomial coefficients to convert our identities into another set of three polynomial identities, which imply Capparelli's partition theorems when the degree of the polynomial tends to infinity. This way we also obtain an interesting new result for the sum of the Capparelli's products. We finish this paper by proposing an infinite hierarchy of polynomial identities.
\end{abstract}

Mathematics Subject Classification. 11B65, 11C08, 11P81, 11P82, 11P83, 11P84, 05A10, 05A15, 05A17.

Keywords. Happy Birthday, Capparelli's partition theorems, $q$-Trinomial coefficients, $q$-Series, Polynomial identities.

\section{Introduction}

George E. Andrews is known for his many accomplishments and impeccable leadership in both his mathematical contributions and in service to the community of mathematics. His influence on research keeps opening new horizons, and at the same time, new doors to young researchers. There are many areas of study he introduced that are now saturated with world-class mathematicians, yet there are many more that the community is only catching up studying. Here, we look at one of these lesser-studied objects: $q$-trinomial coefficients. Introduced by Andrews in collaboration with Baxter, the $q$-trinomial coefficients are defined by

$$
\left(\begin{array}{c}
L, b \\
a
\end{array} ; q\right)_{2}:=\sum_{n \geq 0} q^{n(n+b)} \frac{(q ; q)_{L}}{(q ; q)_{n}(q ; q)_{n+a}(q ; q)_{L-2 n-a}}
$$


where, for any non-negative integer $n,(a ; q)_{n}$ is the standard $q$-Pochhammer symbol [3]:

$$
(a ; q)_{n}:=(1-a)(1-a q)\left(1-a q^{2}\right) \ldots\left(1-a q^{n-1}\right) .
$$

Here and throughout $|q|<1$.

It is easy to verify that

$$
\sum_{a=-L}^{L}\left(\begin{array}{c}
L, b \\
a
\end{array}\right)_{2} t^{a}=\left(t+1+\frac{1}{t}\right)^{L},
$$

which implies the generalized Pascal Triangle for (1.1) with $q=1$ :

$$
\begin{array}{ccccccccccc} 
& & & & 1 & & & & & \\
& & & 1 & 1 & 1 & & & & \\
& & 1 & 2 & 3 & 2 & 1 & & & \\
& 1 & 3 & 6 & 7 & 6 & 3 & 1 & & \\
& 1 & 4 & 10 & 16 & 19 & 16 & 10 & 4 & 1 & \\
. & \vdots & \vdots & \vdots & \vdots & \vdots & \vdots & \vdots & \vdots & \vdots & \ddots
\end{array}
$$

The $q$-trinomial coefficients were studied in $[1,2,4-10,14-17]$. Nevertheless, it appears that the following identities are new.

\section{Theorem 1.1.}

$$
\begin{aligned}
& \sum_{n \geq 0}(-1)^{n} q^{\left(3 n^{2}+n\right) / 2} \frac{\left(q^{3} ; q^{3}\right)_{L}}{(q ; q)_{L-2 n}\left(q^{3} ; q^{3}\right)_{n}} \\
& +q^{2 L+1} \sum_{n \geq 0}(-1)^{n} q^{\left(3 n^{2}-n\right) / 2} \frac{\left(q^{3} ; q^{3}\right)_{L}}{(q ; q)_{L-2 n}\left(q^{3} ; q^{3}\right)_{n}} \\
& =\sum_{j=-L}^{L}\left\{q^{L+j+1}\left(\begin{array}{c}
L, j+1 \\
j
\end{array} ; q^{3}\right)_{2}+q^{L+4 j}\left(\begin{array}{c}
L, j \\
j-1
\end{array} ; q^{3}\right)_{2}\right\} \text {, } \\
& \sum_{n \geq 0}(-1)^{n} q^{\left(3 n^{2}-n\right) / 2} \frac{\left(q^{3} ; q^{3}\right)_{L}}{(q ; q)_{L-2 n}\left(q^{3} ; q^{3}\right)_{n}}=\sum_{j=-L}^{L} q^{2 L-j}\left(\begin{array}{c}
L, j-1 \\
j
\end{array} ; q^{3}\right)_{2} \\
& \sum_{n \geq 0}(-1)^{n} q^{\left(3 n^{2}+n\right) / 2} \frac{\left(q^{3} ; q^{3}\right)_{L}}{(q ; q)_{L-2 n}\left(q^{3} ; q^{3}\right)_{n}}=\sum_{j=-L}^{L} q^{L-j}\left(\begin{array}{c}
L, j \\
j
\end{array} ; q^{3}\right)_{2} \text {. }
\end{aligned}
$$

From the left-hand sides of (1.2)-(1.4), we can easily discover the limiting formulas:

$$
\frac{1}{\left(q ; q^{3}\right)_{\infty}}, \frac{1}{\left(q^{2} ; q^{3}\right)_{\infty}} \text {, and } \frac{1}{\left(q ; q^{3}\right)_{\infty}}
$$

respectively, as $L \rightarrow \infty$ with the aid of the $q$-binomial theorem. However, this is not as easy to see that from the right-hand sides of these identities.

These identities are related to combinatorics and partition theory. As an example, we will show that (1.2) and (1.4) in Theorem 1.1 imply Capparelli's partition theorems. Moreover, (1.3) implies the following new interesting result. 


\section{Theorem 1.2.}

$$
\begin{aligned}
\sum_{m, n \geq 0} \frac{q^{2 m^{2}+6 m n+6 n^{2}-2 m-3 n}}{(q ; q)_{m}\left(q^{3} ; q^{3}\right)_{n}}= & \left(-q^{2},-q^{4} ; q^{6}\right)_{\infty}\left(-q^{3} ; q^{3}\right)_{\infty} \\
& +\left(-q,-q^{5} ; q^{6}\right)_{\infty}\left(-q^{3} ; q^{3}\right)_{\infty} .
\end{aligned}
$$

In Sect. 2, we give a comprehensive list of definitions and identities that will be used in this paper. Section 3 has the proof of Theorem 1.1. This section also includes the dual of these identities and a necessary version of Bailey's lemma for the $q$-trinomial coefficients. We find new polynomial identities that yield Capparelli's partition theorem in Sect. 4. Theorem 1.2, which includes the sum of the two Capparelli's theorem's products, is also proven in Sect. 4. This section also contains a comparison of the mentioned polynomial identities and the previously found polynomial identities [10] that also imply Capparelli's partition theorems. The outlook section, Sect. 5 , includes two new results the authors are planning on presenting soon: a doubly bounded identity involving Warnaar's refinement of the $q$-trinomial coefficients, and also an infinite hierarchy of $q$-series identities.

\section{Necessary Definitions and Identities}

We use the standard notation as in [3]. For formal variables $a_{i}$ and $q$, and a non-negative integer $n$ :

$$
\begin{aligned}
(a ; q)_{\infty} & :=\lim _{n \rightarrow \infty}(a ; q)_{n}, \\
\left(a_{1}, a_{2}, \ldots, a_{k} ; q\right)_{n} & :=\left(a_{1} ; q\right)_{n}\left(a_{2} ; q\right)_{n} \cdots\left(a_{k} ; q\right)_{n} .
\end{aligned}
$$

We can extend the definition of the $q$-Pochhamer symbol to negative $n$ as follows:

$$
(a ; q)_{n}=\frac{(a ; q)_{\infty}}{\left(a q^{n} ; q\right)_{\infty}} .
$$

Observe that (2.1) implies

$$
\frac{1}{(q ; q)_{n}}=0 \quad \text { if } \quad n<0 .
$$

In addition, observe that for non-negative $n$, we have

$$
\left(q^{-1} ; q^{-1}\right)_{n}=(-1)^{n} q^{-\left(\begin{array}{c}
n+1 \\
2
\end{array}\right)}(q ; q)_{n} .
$$

We define the $q$-binomial coefficients in the classical manner as

$$
\left[\begin{array}{c}
m+n \\
m
\end{array}\right]_{q}:= \begin{cases}\frac{(q)_{m+n}}{(q)_{m}(q)_{n}}, & \text { for } m, n \geq 0 \\
0, & \text { otherwise. }\end{cases}
$$

It is well known that for $m \in \mathbb{Z}_{\geq 0}$

$$
\lim _{N \rightarrow \infty}\left[\begin{array}{l}
N \\
m
\end{array}\right]_{q}=\frac{1}{(q ; q)_{m}}
$$


for any $j \in \mathbb{Z}_{\geq 0}$ and $\nu=0$ or 1

$$
\lim _{M \rightarrow \infty}\left[\begin{array}{c}
2 M+\nu \\
M-j
\end{array}\right]_{q}=\frac{1}{(q ; q)_{\infty}} .
$$

We define another $q$-trinomial coefficient for any integer $n$ :

$$
T_{n}\left(\begin{array}{l}
L \\
a
\end{array} ;\right):=q^{(L(L-n)-a(a-n)) / 2}\left(\begin{array}{c}
L, a-n \\
a
\end{array} \frac{1}{q}\right)_{2} .
$$

Theorem 2.1 ( $q$-Binomial theorem). For variables $a, q$, and $z$

$$
\sum_{n \geq 0} \frac{(a ; q)_{n}}{(q ; q)_{n}} z^{n}=\frac{(a z ; q)_{\infty}}{(z ; q)_{\infty}}
$$

In addition, note that the $q$-exponential sum

$$
\sum_{n \geq 0} \frac{q^{n(n-1) / 2}}{(q ; q)_{n}} z^{n}=(-z ; q)_{\infty}
$$

is a limiting case $(a \rightarrow \infty$ after the variable change $z \mapsto-z / a)$ of (2.10).

Another ingredient we will use here is the Jacobi Triple Product Identity $[3]$.

Theorem 2.2 (Jacobi triple product identity).

$$
\sum_{j=-\infty}^{\infty} z^{j} q^{j^{2}}=\left(q^{2},-z q,-\frac{q}{z} ; q^{2}\right)_{\infty}
$$

\section{Proof of Theorem 1.1 and Some $q$-Trinomial Summation Formulas}

We start with the following lemma.

Lemma 3.1. For any integer $n$, we have

$$
\sum_{L \geq 0} \sum_{j=-\infty}^{\infty} \frac{x^{j} t^{L}}{(q ; q)_{L}}\left(\begin{array}{c}
L, j-n \\
j
\end{array} ;\right)_{2}=\frac{\left(t^{2} q^{-n} ; q\right)_{\infty}}{\left(t, \frac{t}{x} q^{-n}, t x ; q\right)_{\infty}}
$$

The $n=0$ case of (3.1) first appeared in the work of Andrews [2, p. 153, (6.6)].

Proof of Lemma 3.1. We start by writing definition (1.1) on the left-hand side of formula (3.1). After a simple cancellation, one sees that the triple sum can be untangled by the change of the summation variables $\nu=k+j$, and $\mu=L-2 k-j$. This change of summation variables, keeping (2.3) in mind, shows that the left-hand side sum of (3.1) can be written as:

$$
\sum_{k \geq 0} \frac{x^{-k} t^{k} q^{-n k}}{(q ; q)_{k}} \sum_{\nu \geq 0} \frac{x^{\nu} t^{\nu} q^{k \nu}}{(q ; q)_{\nu}} \sum_{\mu \geq 0} \frac{t^{\mu}}{(q ; q)_{\mu}}
$$


One can apply the $q$-Binomial Theorem starting from the innermost sum of (3.2). After applying the $q$-binomial Theorem 2.1 with $(a, z)=(0, t)$, and $(a, z)=\left(0, x t q^{k}\right)$, we get

$$
\frac{1}{(t ; q)_{\infty}} \sum_{k \geq 0} \frac{x^{-k} t^{k} q^{-n k}}{(q ; q)_{k}\left(x t q^{k} ; q\right)_{\infty}} .
$$

We rewrite $\left(x t q^{k} ; q\right)_{\infty}$ using $(2.1)$, take the $k$-free portion out of the summation, and use (2.10) once again with $(a, z)=\left(x t, x^{-1} t q^{n}\right)$ to finish the proof.

We can prove Theorem 1.1 using Lemma 3.1.

Proof of Theorem 1.1. Instead of proving these identities directly, we will prove the equality of their generating functions. It is clear that one can prove the equality of the two sides of polynomial identities of the form

$$
A_{L}(q)=B_{L}(q)
$$

by a multi-variable generating function equivalence

$$
\sum_{L \geq 0} \frac{t^{L}}{\left(q^{3} ; q^{3}\right)_{L}} A_{L}(q)=\sum_{L \geq 0} \frac{t^{L}}{\left(q^{3} ; q^{3}\right)_{L}} B_{L}(q) .
$$

On the right-hand side of (3.4) with the choice of $B_{L}(q)$ being the righthand sides of (1.2)-(1.4), we get

$$
\frac{\left(t^{2} q^{2} ; q^{3}\right)_{\infty}}{(t ; q)_{\infty}} \frac{(1+q)}{(1+t q)}, \frac{\left(t^{2} q ; q^{3}\right)_{\infty}}{(t ; q)_{\infty}} \text {, and } \frac{\left(t^{2} q^{2} ; q^{3}\right)_{\infty}}{(t ; q)_{\infty}}
$$

respectively, by Lemma 3.1. Hence, all we need to do is to show that the lefthand side of (3.4) with the choice of $A_{L}(q)$ being the left-hand sides of (1.2)(1.4) yields the same products.

The left-hand side of (1.2) has two sums. The first sum of the left-hand side of (1.2) after being multiplied by $t^{L} /\left(q^{3} ; q^{3}\right)_{L}$, summing over $L$ as suggested in (3.4), and after simple cancellations turns into

$$
\sum_{L, n \geq 0}(-1)^{n} \frac{q^{\frac{3 n^{2}+n}{2}} t^{L}}{(q ; q)_{L-2 n}\left(q^{3} ; q^{3}\right) n} .
$$

We introduce the new summation variable $\nu=L-2 n$. This factors the double sum fully. Keeping (2.3) in mind, we rewrite (3.6) as

$$
\sum_{\nu \geq 0} \frac{t^{\nu}}{(q ; q)_{\nu}} \sum_{n \geq 0} \frac{q^{3 n(n-1) / 2}}{\left(q^{3} ; q^{3}\right)_{n}}\left(-t^{2} q\right)^{n} .
$$

Then, using (2.10) and (2.11) on the two sums, respectively, we see that (3.6) is equal to

$$
\frac{\left(t^{2} q ; q^{3}\right)_{\infty}}{(t ; q)_{\infty}}
$$


The same exact calculation can be done for the second sum on the left-hand side of (1.2), and the left-hand side sums of (1.3) and (1.4). After the simplifications, we see that the products we get from the left-hand side sums after (3.4) is applied to them, are the same as the products (3.5).

In the identities of Theorem 1.1, we replace $q \mapsto 1 / q$, multiply both sides of the equations by $q^{3 L^{2} / 2}$, use (2.4) and (2.9), and do elementary simplifications to get the following theorem.

\section{Theorem 3.2.}

$$
\begin{aligned}
& \sum_{n \geq 0} q^{\left(\begin{array}{c}
L-2 n \\
2
\end{array}\right)} \frac{\left(q^{3} ; q^{3}\right)_{L}}{(q ; q)_{L-2 n}\left(q^{3} ; q^{3}\right)_{n}}+q^{L+1} \sum_{n \geq 0} q_{2}^{\left(\begin{array}{c}
L-2 n+1 \\
2
\end{array}\right)+n} \frac{\left(q^{3} ; q^{3}\right)_{L}}{(q ; q)_{L-2 n}\left(q^{3} ; q^{3}\right)_{n}} \\
& =\sum_{j=-L}^{L} q^{\frac{3 j^{2}+j}{2}}\left\{T_{-1}\left(\begin{array}{c}
L \\
j
\end{array} q^{3}\right)+T_{-1}\left(\begin{array}{c}
L \\
j+1
\end{array} ; q^{3}\right)\right\} \\
& \sum_{n \geq 0} q^{\left(\begin{array}{c}
L-2 n \\
2
\end{array}\right)} \frac{\left(q^{3} ; q^{3}\right)_{L}}{(q ; q)_{L-2 n}\left(q^{3} ; q^{3}\right)_{n}}=\sum_{j=-L}^{L} q^{\frac{3 j^{2}-j}{2}} T_{1}\left(\begin{array}{c}
L \\
j
\end{array} ; q^{3}\right) \\
& \sum_{n \geq 0} q^{\frac{(L-2 n)^{2}}{2}} \frac{\left(q^{3} ; q^{3}\right)_{L}}{(q ; q)_{L-2 n}\left(q^{3} ; q^{3}\right)_{n}}=\sum_{j=-L}^{L} q^{\frac{3 j^{2}+2 j}{2}} T_{0}\left(\begin{array}{c}
L \\
j
\end{array} ; q^{3}\right)
\end{aligned}
$$

Building on the development in [7,9,14], Warnaar [15, eqs. (10), (14)] proved the following summation formulas.

Theorem 3.3 (Warnaar).

$$
\begin{gathered}
\sum_{i \geq 0} q^{\frac{i^{2}}{2}}\left[\begin{array}{c}
L \\
i
\end{array}\right]_{q} T_{0}\left(\begin{array}{l}
i \\
a
\end{array} ; q\right)=q^{\frac{a^{2}}{2}}\left[\begin{array}{c}
2 L \\
L-a
\end{array}\right]_{q} \\
\sum_{i \geq 0} q^{\left(\begin{array}{c}
i \\
2
\end{array}\right)}\left(1+q^{L}\right)\left[\begin{array}{c}
L \\
i
\end{array}\right]_{q} T_{1}\left(\begin{array}{l}
i \\
a
\end{array} ; q\right)=\left(1+q^{a}\right) q^{\left(\begin{array}{c}
a \\
2
\end{array}\right)}\left[\begin{array}{c}
2 L \\
L-a
\end{array}\right]_{q}
\end{gathered}
$$

We found a similar new summation formula:

\section{Theorem 3.4.}

$$
\sum_{i \geq 0} q^{\left(\begin{array}{c}
i+1 \\
2
\end{array}\right)}\left[\begin{array}{c}
L \\
i
\end{array}\right]_{q}\left\{T_{-1}\left(\begin{array}{c}
i \\
a
\end{array} ; q\right)+T_{-1}\left(\begin{array}{c}
i \\
a+1
\end{array} ; q\right)\right\}=q^{\left(\begin{array}{c}
a+1 \\
2
\end{array}\right)}\left[\begin{array}{c}
2 L+1 \\
L-a
\end{array}\right]_{q} .
$$

Proof. To prove (3.12), we need the following identity of Berkovich-McCoyOrrick [8, p. 815, (4.8)]:

$$
\begin{aligned}
& T_{-1}\left(\begin{array}{c}
L \\
a
\end{array} ; q\right)+T_{-1}\left(\begin{array}{c}
L \\
a+1
\end{array} ; q\right) \\
& \quad=\frac{1}{1-q^{L+1}}\left\{T_{1}\left(\begin{array}{c}
L+1 \\
a
\end{array} ; q\right)-q^{(L+1-a) / 2} T_{0}\left(\begin{array}{c}
L+1 \\
a
\end{array} ; q\right)\right\}
\end{aligned}
$$


After the use of (3.13) on the left-hand side of (3.12), we employ

$$
\frac{1}{1-q^{i+1}}\left[\begin{array}{c}
L \\
i
\end{array}\right]_{q}=\frac{1}{1-q^{L+1}}\left[\begin{array}{c}
L+1 \\
i+1
\end{array}\right]_{q},
$$

and summations (3.10) and (3.11). This yields the right-hand side of (3.12) after some elementary simplifications.

Theorem 3.5. Let $F_{j}(L)$ and $\alpha_{j}(a)$ be sequences, depending on $L$ and $a$, respectively, for $j=-1,0$ or 1 . If

$$
\begin{aligned}
F_{0}(L) & =\sum_{a=-\infty}^{\infty} \alpha_{0}(a) T_{0}\left(\begin{array}{c}
L \\
a
\end{array} ; q\right) \\
F_{1}(L) & =\sum_{a=-\infty}^{\infty} \alpha_{1}(a) T_{1}\left(\begin{array}{c}
L \\
a
\end{array} ; q\right) \\
F_{-1}(L) & =\sum_{a=-\infty}^{\infty} \alpha_{-1}(a)\left\{T_{-1}\left(\begin{array}{l}
L \\
a
\end{array} ; q\right)+T_{-1}\left(\begin{array}{c}
L \\
a+1
\end{array} ; q\right)\right\}
\end{aligned}
$$

hold, then

$$
\begin{aligned}
\sum_{i \geq 0} q^{\frac{i^{2}}{2}}\left[\begin{array}{c}
L \\
i
\end{array}\right]_{q} F_{0}(i) & =\sum_{a=-\infty}^{\infty} \alpha_{0}(a) q^{\frac{a^{2}}{2}}\left[\begin{array}{c}
2 L \\
L-a
\end{array}\right]_{q} \\
\left(1+q^{L}\right) \sum_{i \geq 0} q^{\left(\begin{array}{c}
i \\
2
\end{array}\right)}\left[\begin{array}{c}
L \\
i
\end{array}\right]_{q} F_{1}(i) & =\sum_{a=-\infty}^{\infty} \alpha_{1}(a)\left(1+q^{a}\right) q^{\left(\begin{array}{c}
a \\
2
\end{array}\right)}\left[\begin{array}{c}
2 L \\
L-a
\end{array}\right]_{q} \\
\sum_{i \geq 0} q^{\left(\begin{array}{c}
i+1 \\
2
\end{array}\right)}\left[\begin{array}{c}
L \\
i
\end{array}\right]_{q} F_{-1}(i) & =\sum_{a=-\infty}^{\infty} \alpha_{-1}(a) q^{\left(\begin{array}{c}
a+1 \\
2
\end{array}\right)}\left[\begin{array}{c}
2 L+1 \\
L-a
\end{array}\right]_{q}
\end{aligned}
$$

are true.

Proof. We apply (3.10)-(3.12) to (3.15)-(3.17) and get (3.18)-(3.20), respectively.

\section{New Polynomial Identities Implying Capparelli's Partition Theorems}

We apply (3.18) to (3.9) to get

$$
\sum_{L, n \geq 0} q^{\frac{(L-2 n)^{2}+3 L^{2}}{2}} \frac{\left(q^{3} ; q^{3}\right)_{M}}{(q ; q)_{L-2 n}\left(q^{3} ; q^{3}\right)_{n}\left(q^{3} ; q^{3}\right)_{M-L}}=\sum_{j=-M}^{M} q^{3 j^{2}+j}\left[\begin{array}{c}
2 M \\
M+j
\end{array}\right]_{q^{3}}
$$

We introduce the new variable $m=L-2 n$, and let

$$
Q(m, n):=2 m^{2}+6 m n+6 n^{2},
$$

and observe that

$$
Q(m, n)=\frac{(L-2 n)^{2}+3 L^{2}}{2}
$$


after the change of variable. Hence, (4.1) can be written as follows.

\section{Theorem 4.1.}

$$
\sum_{m, n \geq 0} \frac{q^{Q(m, n)}\left(q^{3} ; q^{3}\right)_{M}}{(q ; q)_{m}\left(q^{3} ; q^{3}\right)_{n}\left(q^{3} ; q^{3}\right)_{M-2 n-m}}=\sum_{j=-M}^{M} q^{3 j^{2}+j}\left[\begin{array}{c}
2 M \\
M+j
\end{array}\right]_{q^{3}} .
$$

Recall that (4.2) is Theorem 7.1 in [10]. Letting $M \rightarrow \infty$ in (4.2), using (2.8), and the Jacobi Triple Product Identity (2.12) on the right-hand side, we obtain the following.

\section{Theorem 4.2.}

$$
\sum_{m, n \geq 0} \frac{q^{Q(m, n)}}{(q ; q)_{m}\left(q^{3} ; q^{3}\right)_{n}}=\left(-q^{2},-q^{4} ; q^{6}\right)_{\infty}\left(-q^{3} ; q^{3}\right)_{\infty}
$$

The authors recently discovered another polynomial identity [10, Theorem 1.3, (1.12)] that implies the same $q$-series identity (4.3) as $N \rightarrow \infty$ :

Theorem 4.3. For any non-negative integer $N$, we have

$$
\begin{aligned}
& \sum_{m, n \geq 0} q^{Q(m, n)}\left[\begin{array}{c}
3(N-2 n-m) \\
m
\end{array}\right]_{q}\left[\begin{array}{c}
2(N-2 n-m)+n \\
n
\end{array}\right]_{q^{3}} \\
& =\sum_{l=0}^{N} q^{3\left(\begin{array}{c}
N-2 l \\
2
\end{array}\right)}\left[\begin{array}{l}
N \\
2 l
\end{array}\right]_{q^{3}}\left(-q^{2},-q^{4} ; q^{6}\right)_{l} .
\end{aligned}
$$

The identity (4.3) was independently proposed by Kanade-Russell [12] and Kurşungöz [13]. They showed that (4.3) is equivalent to the following partition theorem.

Theorem 4.4 (Capparelli's first partition theorem [11]). For any integer $n$, the number of partitions of $n$ into distinct parts where no part is congruent to \pm 1 modulo 6 is equal to the number of partitions of $n$ into parts, not equal to 1 , where the minimal difference between consecutive parts is 2. In fact, the difference between consecutive parts is greater than or equal to 4 unless consecutive parts are $3 k$ and $3 k+3$ (yielding a difference of 3 ), or $3 k-1$ and $3 k+1$ (yielding a difference of 2 ) for some $k \in \mathbb{N}$.

Theorem 4.4 was first proven by Andrews in [2].

Analogously, we apply (3.19) to (3.8). This way we are led to the theorem:

\section{Theorem 4.5.}

$$
\begin{gathered}
\sum_{m, n \geq 0} \frac{q^{Q(m, n)-2 m-3 n}\left(q^{3} ; q^{3}\right)_{M}}{(q ; q)_{m}\left(q^{3} ; q^{3}\right)_{n}\left(q^{3} ; q^{3}\right)_{M-2 n-m}}\left(1+q^{3 M}\right) \\
\quad=\sum_{j=-M}^{M} q^{3 j^{2}-2 j}\left(1+q^{3 j}\right)\left[\begin{array}{c}
2 M \\
M+j
\end{array}\right]_{q^{3}} .
\end{gathered}
$$


Letting $M$ tend to infinity, and using (2.8) and (2.12) on the right-hand side proves Theorem 1.2.

Similar to the above calculations, we apply (3.20) to (3.7) and get the theorem:

\section{Theorem 4.6.}

$$
\begin{aligned}
& \sum_{m, n \geq 0} \frac{q^{Q(m, n)+m+3 n}\left(q^{3} ; q^{3}\right)_{M}}{(q ; q)_{m}\left(q^{3} ; q^{3}\right)_{n}\left(q^{3} ; q^{3}\right)_{M-2 n-m}}+\sum_{m, n \geq 0} \frac{q^{Q(m, n)+3 m+6 n+1}\left(q^{3} ; q^{3}\right)_{M}}{(q ; q)_{m}\left(q^{3} ; q^{3}\right)_{n}\left(q^{3} ; q^{3}\right)_{M-2 n-m}} \\
& \quad=\sum_{j=-M-1}^{M} q^{3 j^{2}+2 j\left[\begin{array}{c}
2 M+1 \\
M-j
\end{array}\right]_{q^{3}} .}
\end{aligned}
$$

Letting $M \rightarrow \infty$ and using (2.8) and (2.12) on the right-hand side, we get the result:

\section{Theorem 4.7.}

$$
\sum_{m, n \geq 0} \frac{q^{Q(m, n)+m+3 n}}{(q ; q)_{m}\left(q^{3} ; q^{3}\right)_{n}}+\sum_{m, n \geq 0} \frac{q^{Q(m, n)+3 m+6 n+1}}{(q ; q)_{m}\left(q^{3} ; q^{3}\right)_{n}}=\left(-q,-q^{5} ; q^{6}\right)_{\infty}\left(-q^{3} ; q^{3}\right)_{\infty} \text {. }
$$

It is instructive to compare (4.5) with the following polynomial identity $[10$, Theorem 1.3, (1.12)], which also implies (4.6) as $N \rightarrow \infty$ :

Theorem 4.8. For any non-negative integer $N$, we have

$$
\begin{aligned}
& \sum_{m, n \geq 0} q^{Q(m, n)+m+3 n}\left[\begin{array}{c}
3(N-2 n-m)+2 \\
m
\end{array}\right]_{q}\left[\begin{array}{c}
2(N-2 n-m)+n+1 \\
n
\end{array}\right]_{q^{3}} \\
& +\sum_{m, n \geq 0} q^{Q(m, n)+3 m+6 n+1}\left[\begin{array}{c}
3(N-2 n-m) \\
m
\end{array}\right]_{q}\left[\begin{array}{c}
2(N-2 n-m)+n \\
n
\end{array}\right]_{q^{3}} \\
& =\sum_{l=0}^{N} q^{3\left(\begin{array}{c}
N-2 l \\
2
\end{array}\right)}\left[\begin{array}{c}
N+1 \\
2 l+1
\end{array}\right]_{q^{3}}\left(-q ; q^{6}\right)_{l+1}\left(-q^{5} ; q^{6}\right)_{l} .
\end{aligned}
$$

We note that (4.6) first appeared in Kurşungöz [13]. In fact, this is equivalent to the Cappareli's second partition theorem.

Theorem 4.9 (Capparelli's second partition theorem [11]). For any integer $n$, the number of partitions of $n$ into distinct parts, where no part is congruent to \pm 2 modulo 6 is equal to the number of partitions of $n$ into parts, not equal to 2, where the minimal difference between consecutive parts is 2. In fact, the difference between consecutive parts is greater than or equal to 4 unless consecutive parts are $3 k$ and $3 k+3$ (yielding a difference of 3 ), or $3 k-1$ and $3 k+1$ (yielding a difference of 2 ) for some $k \in \mathbb{N}$.

We note that Kurşungöz [13] showed the equivalence of (4.6) to Theorem 4.9. On the other hand, Kanade-Russell [12] showed the equivalence of a slightly different (yet equivalent) double sum identity to the Capparelli's Second Partition Theorem. 
Comparing Theorems 4.1 and 4.3, and Theorems 4.6 and 4.8, we see that the identities proven here look somewhat simpler. On the other hand, the objects that appear on both sides of the identities from [10] clearly come with combinatorial interpretations and are made up of objects with manifestly positive coefficients. It is not necessarily clear that the left-hand sides of (4.2), (4.4), and (4.5) have positive coefficients at first sight. A combinatorial study of these objects as generating functions, which would also show the non-negativity of the coefficients of these polynomials, is a task for the future.

\section{Outlook}

Identity (3.9) is the special case $M \rightarrow \infty$ of the following doubly bounded identity.

\section{Theorem 5.1.}

$$
\sum_{\substack{m \geq 0, L \equiv m(\bmod 2)}} q^{\frac{m^{2}}{2}}\left[\begin{array}{c}
3 M \\
m
\end{array}\right]_{q}\left[\begin{array}{c}
2 M+\frac{L-m}{2} \\
2 M
\end{array}\right]_{q^{3}}=\sum_{j=-\infty}^{\infty} q^{\frac{3 j^{2}+2 j}{2}} \mathcal{T}\left(\begin{array}{c}
L, M \\
j, j
\end{array} ; q^{3}\right),
$$

where

$$
\mathcal{T}\left(\begin{array}{c}
L, M \\
a, b
\end{array} ; q\right):=\sum_{\substack{n \geq 0, L-a \equiv n(\bmod 2)}} q^{\frac{n^{2}}{2}}\left[\begin{array}{c}
M \\
n
\end{array}\right]_{q}\left[\begin{array}{c}
M+b+\frac{L-a-n}{2} \\
M+b
\end{array}\right]_{q}\left[\begin{array}{c}
M-b+\frac{L+a-n}{2} \\
M-b
\end{array}\right]_{q} .
$$

The refinement (5.2) of the $q$-trinomial coefficients was first introduced by Warnaar $[16,17]$.

In the forthcoming paper, we will show that Theorem 5.1 implies the following infinite hierarchy of identities.

Theorem 5.2. Let $\nu$ be a positive integer, and let $N_{k}=n_{k}+n_{k+1}+\cdots+n_{\nu}$, for $k=1,2, \ldots, \nu$. Then

$$
\begin{aligned}
& \sum_{\substack{i, m, n_{1}, n_{2}, \ldots, n_{\nu} \geq 0, i+m \equiv N_{1}+N_{2}+\cdots+N_{\nu}(\bmod 2)}} q^{\frac{m^{2}+3\left(i^{2}+N_{1}^{2}+N_{2}^{2}+\cdots+N_{\nu}^{2}\right)}{2}}\left[\begin{array}{c}
L-N_{1} \\
i
\end{array}\right]_{q^{3}}\left[\begin{array}{c}
3 n_{\nu} \\
m
\end{array}\right]_{q} \\
& \times\left[\begin{array}{c}
2 n_{\nu}+\left(i-N_{1}-N_{2}-\cdots-N_{\nu}-m\right) / 2 \\
2 n_{\nu}
\end{array} \prod_{q^{3}}^{\nu-1}\left[\begin{array}{c}
i-\sum_{k=1}^{j} N_{k}+n_{j} \\
n_{j}
\end{array}\right]_{q^{3}}\right. \\
& =\sum_{j=\infty}^{\infty} q^{3\left(\begin{array}{c}
\nu+2 \\
2
\end{array}\right) j^{2}+j}\left(\begin{array}{c}
L,(\nu+2) j \\
(\nu+2) j
\end{array} ; q^{3}\right)_{2} .
\end{aligned}
$$

\section{Acknowledgements}

Open access funding provided by Johannes Kepler University Linz. The authors would like to thank the Algorithmic Combinatorics group of the Research Institute for Symbolic Computation, led by Peter Paule, for their hospitality 
and for providing them the research environment, where this work has flourished. Research of Alexander Berkovich is partly supported by the Simons Foundation, Award ID: 308929. Research of Ali Kemal Uncu is supported by the Austrian Science Fund FWF, SFB50-07 and SFB50-09 Projects. We would like to thank Chris Jennings-Shaffer for his careful reading of the manuscript.

Open Access. This article is distributed under the terms of the Creative Commons Attribution 4.0 International License (http://creativecommons.org/licenses/ by/4.0/), which permits unrestricted use, distribution, and reproduction in any medium, provided you give appropriate credit to the original author(s) and the source, provide a link to the Creative Commons license, and indicate if changes were made.

Publisher's Note Springer Nature remains neutral with regard to jurisdictional claims in published maps and institutional affiliations.

\section{References}

[1] Andrews, G.E.: Euler's "exemplum memorabile inductionis fallacis" and $q$ trinomial coefficients. J. Amer. Math. Soc. 3(3), 653-669 (1990)

[2] Andrews, G.E.: Schur's theorem, Capparelli's conjecture and $q$-trinomial coefficients. In: Andrews, G.E., Bressoud, D.M., Parson, L.A. (eds.) The Rademacher Legacy to Mathematics (University Park, PA, 1992), Contemp. Math., 166, pp. 141-154. Amer. Math. Soc., Providence, RI (1994)

[3] Andrews, G.E.: The Theory of Partitions. Cambridge University Press, Cambridge (1998)

[4] Andrews, G.E.: Rogers-Ramanujan polynomials for modulus 6. In: Berndt, B.C., Diamond, H.G., Hildebrand, A.J. (eds.) Analytic Number Theory, Vol. 1 (Allerton Park, IL, 1995), Progr. Math., 138, pp. 17-30. Birkhäuser Boston, Boston, MA (1996)

[5] Andrews, G.E.: $q$-Trinomial coefficients and Rogers-Ramanujan type identities. In: Berndt, B.C., Diamond, H.G., Halberstam, H., Hildebrand, A. (eds.) Analytic Number Theory (Allerton Park, IL, 1989), Progr. Math., 85, pp. 1-11. Birkhäuser Boston, Boston, MA (1990)

[6] Andrews, G.E., Baxter, R.J.: Lattice gas generalization of the hard hexagon model. III. $q$-trinomial coefficients. J. Statist. Phys. 47(3-4), 297-330 (1987)

[7] Andrews, G.E., Berkovich, A.: A trinomial analogue of Bailey's lemma and $N=$ 2 superconformal invariance. Comm. Math. Phys. 192(2), 245-260 (1998)

[8] Berkovich, A., McCoy, B.M., Orrick, W.P.: Polynomial identities, indices, and duality for the $N=1$ superconformal model $S M(2,4 \nu)$. J. Statist. Phys. 83(5-6), 795-837 (1996) 
[9] Berkovich, A., McCoy, B.M., Pearce, P.A.: The perturbations $\phi_{2,1}$ and $\phi_{1,5}$ of the minimal models $M\left(p, p^{\prime}\right)$ and the trinomial analogue of Bailey's lemma. Nuclear Phys. B 519(3), 597-625 (1998)

[10] Berkovich, A., Uncu, A.K.: Polynomial identities implying Capparelli's partition theorems. J. Number Theory (2019). https://doi.org/10.1016/j.jnt.2019.02.028

[11] Capparelli, S.: A combinatorial proof of a partition identity related to the level 3 representations of a twisted affine Lie algebra. Comm. Algebra 23(8), 2959-2969 (1995)

[12] Kanade, S., Russell, M.C.: Staircases to analytic sum-sides for many new integer partition identities of Rogers-Ramanujan type. Electron. J. Combin. 26(1), \#P1.6 (2019)

[13] Kurşungöz, K.: Andrews-Gordon type series for Capparelli's and GöllnitzGordon identities. J. Combin. Theory Ser. A 165, 117-138 (2019)

[14] Warnaar, S.O.: A note on the trinomial analogue of Bailey's lemma. J. Combin. Theory Ser. A 81(1), 114-119 (1998)

[15] Warnaar, S.O.: q-Trinomial identities. J. Math. Phys. 40(5), 2514-2530 (1999)

[16] Warnaar, S.O.: Refined $q$-trinomial coefficients and character identities. J. Statist. Phys. 102(3-4), 1065-1081 (2001)

[17] Warnaar, S.O.: The generalized Borwein conjecture. II. Refined $q$-trinomial coefficients. Discrete Math. 272(2-3) (2003)

Alexander Berkovich

Department of Mathematics

University of Florida

358 Little Hall

Gainesville

FL 32611

USA

e-mail: alexb@ufl.edu

Ali Kemal Uncu

Johann Radon Institute for Computational and Applied Mathematics (RICAM)

Austrian Academy of Sciences (OEAW)

Altenbergerstrasse 69

4040 Linz

Austria

e-mail: akuncu@risc.jku.at;

akuncu@ricam.oeaw.ac.at

Received: 25 October 2018.

Accepted: 16 May 2019. 